Check for updates

Cite this: RSC Adv., 2018, 8, 33464

\title{
The CRISPR-Cas9 system: a promising tool for discovering potential approaches to overcome drug resistance in cancer
}

\author{
Jiayu Zhang, Wenlong Zhou, Xiaoxuan Wang and Lihui Wang (D) *
}

The CRISPR-Cas system was identified in bacteria as an immune defense mechanism against threats from the external environment. A common form of this system, called CRISPR-Cas9, is now widely used in gene editing, especially in mammalian cells. Through CRISPR-Cas9, gene knock-ins or knock-outs have become more feasible, thus deepening our understanding of the mechanisms of human diseases, including cancers, and suggesting possible treatment strategies. In this review, we discuss how CRISPR-Cas 9 can be used as a tool to discover more about drug-resistance in cancers, including both the underlying mechanisms and ways to overcome them.

Received 27th May 2018

Accepted 18th September 2018

DOI: $10.1039 / \mathrm{c} 8 \mathrm{ra04509g}$

rsc.li/rsc-advances

ered.' Subsequently, scientists put forward the idea that the CRISPR-Cas system is a potential method for RNAprogrammable genome editing. ${ }^{8}$ The successful applications of the CRISPR-Cas system in mammalian cells demonstrate the easy programmability and wide applicability of this technology. ${ }^{9}$ In 2015, it was shown that CRISPR-Cas9 controls the cleavage of target DNA. ${ }^{10}$ Knight et al. found that Cas9 protein can successfully navigate the chromatin structure of mammalian genomes. ${ }^{11}$ In mammalian cells, the CRISPR-Cas9 system is largely divided into two parts: the chimeric single guide RNA (gRNA) and the Cas9 nuclease protein. The gRNA targets the $20 \mathrm{bp}$ nucleotide region next to a short DNA sequence called the protospacer-adjacent motif (PAM). The gRNA then directs the Cas9 protein to the position of the target sequence. Cas9 protein has two domains with endonuclease activity, HNH and RuvC, which cut the two strands of the target gene. ${ }^{12}$ After the DNA is cleaved, cells attempt to repair the break using the non-homologous end joining (NHEJ) or homologous recombination (HR) methods, and this eventually achieves the goal of gene editing. ${ }^{13}$ Consequently, the CRISPRCas9 system has become a current research hotspot.

Department of Pharmacology, Shenyang Pharmaceutical University, 110016 Shenyang, PR China. E-mail: lhwang@syphu.edu.cn

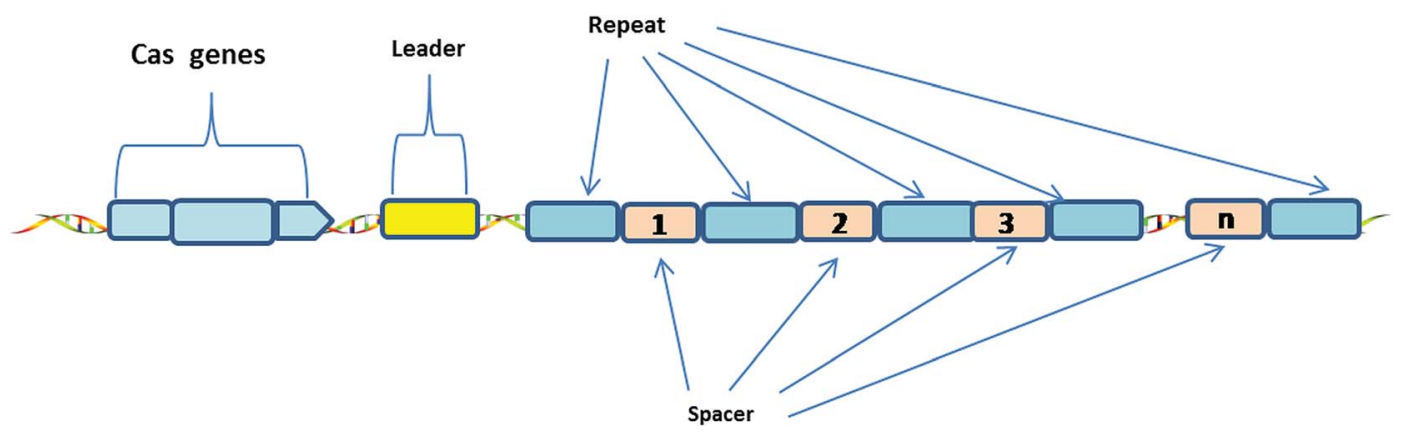

Fig. 1 Generic Type I CRISPR locus in prokaryotes. 
A CRISPR locus consists of Clustered Regularly Interspaced Short Palindromic Repeats (continuous 24-47 bp repeats), which are separated by unique spacer segments (integrated foreign DNA sequences, 30-40 bp in length), and CRISPRassociated (Cas) genes, which surround the CRISPR locus and encode Cas enzymes. The leader sequence (a conserved sequence of several hundred base pairs) acts as a promoter for transcription of the CRISPR array. ${ }^{1}$

\subsection{Mechanism of CRISPR interference}

As the research moved along, CRISPR immunity was divided into three phases (Fig. 2).${ }^{14}$ Firstly, during the adaptation stage, the CRISPR system will gain new spacers by integrating a portion of the foreign genome into the CRISPR sequence. Secondly, the CRISPR array will be transcribed to produce small guide RNAs, also known as CRISPR RNAs (crRNAs). Finally, the crRNA guides the interference and cuts the foreign genes by way of homology-dependent cleavage to achieve silencing of the target DNA. ${ }^{15}$ mature crRNAs; (3) the effector complex combines with foreign genetic elements to interfere with them and degrade them.

\subsection{Biological function of the CRISPR-Cas system}

1.2.1 Immunity function and endogenous regulation. The combination of CRISPR sequences and Cas proteins provides bacteria with their own adaptive immune system which can resist interference from external nucleic acid. ${ }^{16,17}$ Furthermore, the CRISPR-Cas system plays a fundamental role in endogenous regulation of gene expression. A previous study suggested that the CRISPR-Cas system was associated with lysogenic modifications of Pseudomonas aeruginosa, including reduced biofilm formation. ${ }^{18}$ Development of the fruiting body of Myxococcus xanthus was also related to the CRISPR-Cas system. ${ }^{19}$

1.2.2 Genome evolution and DNA repair. The CRISPR-Cas system also has positive effects on genome evolution by selftargeting. CRISPR-Cas-mediated DNA cleavage can potentially lead to large-scale genome rearrangements. Previous research has shown that the repetitive CRISPR sequences play active roles in the process of genome rearrangements. ${ }^{20}$ Cells with the

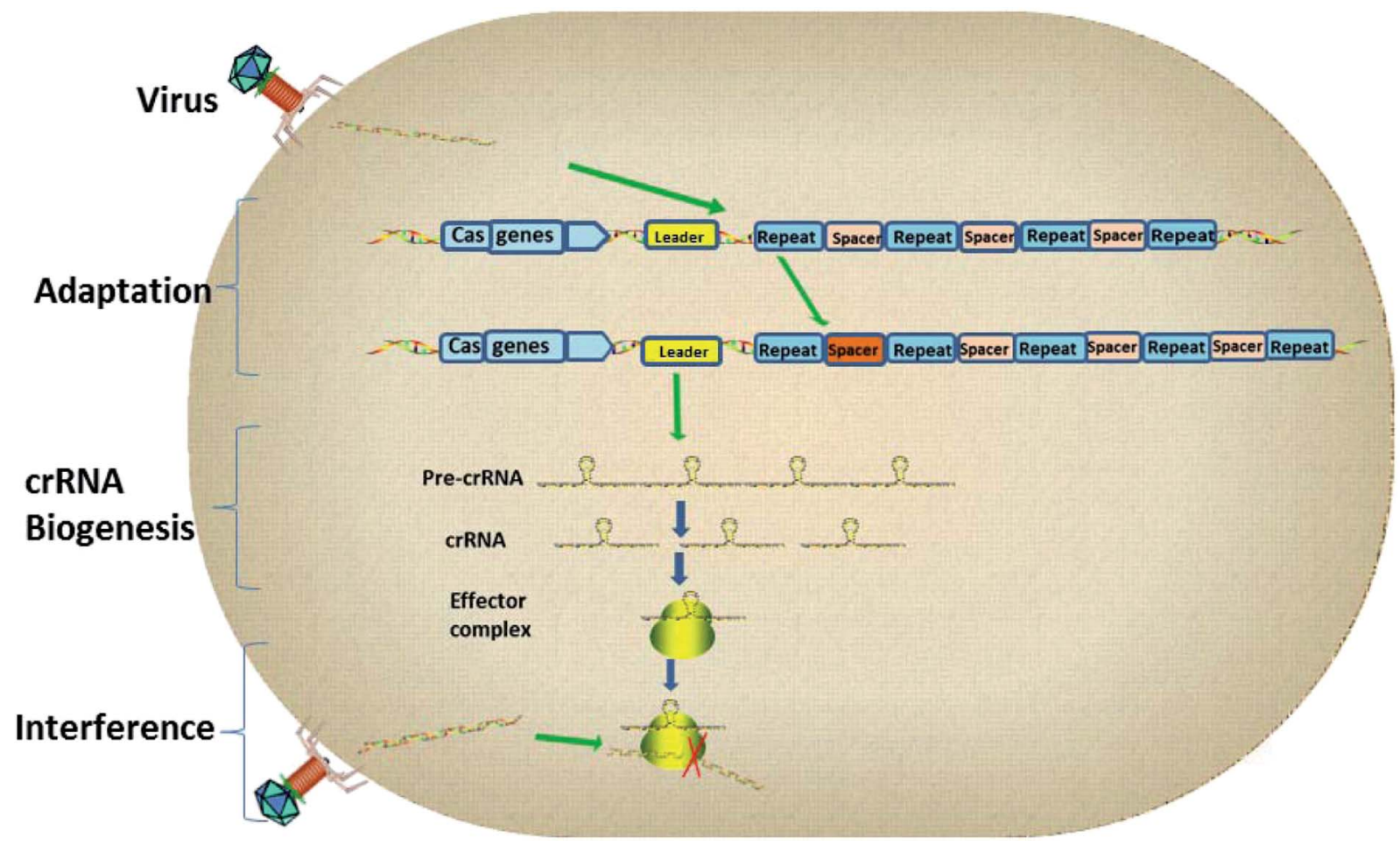

Fig. 2 The three stages of CRISPR interference.

CRISPR loci contain clusters of repeats and spacers that are flanked by a leader sequence and Cas genes. The three stages of CRISPR immunity are as follows: (1) the CRISPR system gains new spacers by integrating a portion of the foreign plasmid or virus genome into the CRISPR sequence; (2) transcription of the CRISPR array generates pre-crRNA, which is processed to give
CRISPR-Cas system will improve their adaptive ability. In other words, the CRISPR system is related to evolution. Furthermore, this system not only defends against exogenous elements and viral infections, ${ }^{21}$ but also has DNA repair functions. For example, strains lacking the Cas1 protein are more sensitive to DNA damage, and chromosomal segregation in these strains is 
impaired. CRISPR-deficient strains also display these phenotypes.

\subsection{The differences between CRISPR and other gene editing techniques}

1.3.1 Zinc finger endonucleases (ZFNs). Zinc finger endonucleases are considered to be the first generation of artificial endonuclease. The zinc finger is a special kind of protein motif that binds to DNA. Artificial endonucleases were created by linking two different zinc finger motifs to the cleavage domain of the Fok I restriction enzyme. ${ }^{22}$ The ZFN identifies the target sequence and the FokI cleavage domain cuts the DNA. This method targets a specific DNA sequence by using a ZFN to change the recognition properties of the DNA-binding domain ${ }^{22}$ (see Fig. 3b).

1.3.2 Transcription activator-like effector nucleases (TALENs). TALENs, the second generation of artificial endonuclease, identify the target DNA sequence using a transcription activator-like effector (TALE), and then cut the DNA at a specific site to form a double-stranded break (DSB). Multiple bases are deleted or inserted to change the targeted sequences with the help of NHEJ or HR. Apparently, TALENs are similar to ZFNs. They consist of specific DNA-binding regions and nonspecific DNA-cleavage regions, which alter gene sequences by inducing DNA breaks ${ }^{23}$ (see Fig. 3c). However, ZFNs or TALENs tools require the synthesis of a large number of guide proteins. In addition, TALEN is also more difficult to be set up in a general laboratory than CRISPR. ${ }^{24,25}$

1.3.3 RNA interference (RNAi). RNA interference (RNAi) is also a commonly used gene silencing method. It is a posttranscriptional gene silencing process mediated by doublestranded RNA molecules, ${ }^{26}$ the dsRNA molecules enter the cells and specifically degrade the homologous mRNA, thereby specifically and efficiently inhibiting the expression of the corresponding genes ${ }^{27}$ (see Fig. 3d). RNAi usually involves small RNAs such as SiRNA, shRNA and miRNA. This method has been widely used in the research of gene function and gene expression regulation mechanisms. However, CRISPR-Cas9 is superior to this method. First, compared to RNA interference technology, CRISPR-Cas9 technology can permanently down-regulate the target genes and it provides an effective gene therapy. ${ }^{28}$ Second, CRISPR-Cas9 technology is not limited by the type of cell lines, it can detect pathogenic genes that RNA interference cannot found. ${ }^{29}$ Third, RNAi is sometimes difficult to interpret phenotypic changes, and the knockdown in gene expression by siRNA sometimes cannot alter the gene phenotype, it will retain gene function. ${ }^{30}$

\section{Revealing the underlying mechanisms in drug resistance with CRISPR-Cas9}

Drug resistance, especially acquired resistance, is the most common problem in long-term drug treatment. Usually, the emergence of drug resistance is a slow and gradual process, suggesting the gene manipulation tool to explore the underlying mechanisms of drug resistance should be a more stable. Compared to RNA interference, CRISPR-Cas9 can permanently remove the interesting genes. Moreover, gene mutation is considered as one of the important reason to induce drug resistance in cancer. In contrast to ZFNs and TALENs technologies, CRISPR-Cas9 is easy to operate and can knock in multiple gene mutations simultaneously, which greatly improves the experimental efficiency. Therefore, accompany with the coming of CRISPR-Cas9 era, the investigation of drug resistance in cancers also made a great progression.

\subsection{ARID1A in HER2-targeting antibody resistance}

HER2-targeting antibody, such as trastuzumab, is an efficacy drug for treatment with breast cancers with HER2 positive expression. However, resistance to trastuzumab reduces the long-term efficacy of this treatment, and it is therefore important to reveal the underlying resistance mechanism. Berns et al. found that directly targeting the PI3K/mTOR pathway seems to be the key to reversing the drug resistance. Recurrent inactivating mutations in ARID1A have been identified at high frequencies in human cancers. ${ }^{31,32}$ Loss of ARID1A activates Annexin A1 (ANXA1), a member of the annexin calcium/ phospholipid-binding protein family which is involved in cell proliferation and tumor progression, ${ }^{33}$ then activates AKT in the HER2/PI3K/mTOR pathway, which finally produces drug resistance. It was verified that completely knocking out ARID1A in BT474 cells by the CRISPR-Cas9-based genome editing technique activated the expression of ANXA1, and high expression of ANXA1 increased the resistance to trastuzumab. ${ }^{34}$ The above results indicate that ANXA1 might be a novel target for treatment with HER2-targeting antibody resistance.

\subsection{Rpn13 and CXCR in proteasome inhibitors resistance}

Proteasome inhibitors, such as bortezomib, is used clinically for multiple myeloma (MM) therapy, ${ }^{35}$ but the development of drug resistance limits its long-term efficacy. Song et al. demonstrated that the expression of the proteasome-related ubiquitination receptor Rpn13 in MM cells was higher than that in normal blood cells. The function of Rpn13 in MM was confirmed by knockout Rpn13 using CRISPR-Cas9 technology. They found Rpn13 would meditated cell proliferation and contributed to further study showed that knockout of Rpn13 by CRISPR-Cas 9 would contributed to bortezomib resistance, suggesting targeting Rpn13 might be a novel approach to overcome proteasome inhibitor resistance. ${ }^{36}$ Recently, another group also demonstrated the role of $\mathrm{CXCR} / \mathrm{SDF}-1$ signaling pathway in bortezomib resistance. Chen et al. used the CRISPR-Cas9 system to knockout the CXCR gene, and found CXCR4 silencing in MCL cells led to a significant reduction in proliferation, cell adhesion, and colony formation. Furthermore, they elucidated a novel bortezomib resistance mechanism which is triggered by ROS release, following by CXCR/SDF-1 pathway activation, next resulted in autophagy increasing, and last induced drug resistance. ${ }^{37}$ This example shows that the CRISPRCas9 system can improve our understanding of the pathways which are involved in cancer, and then provide new means for 
a

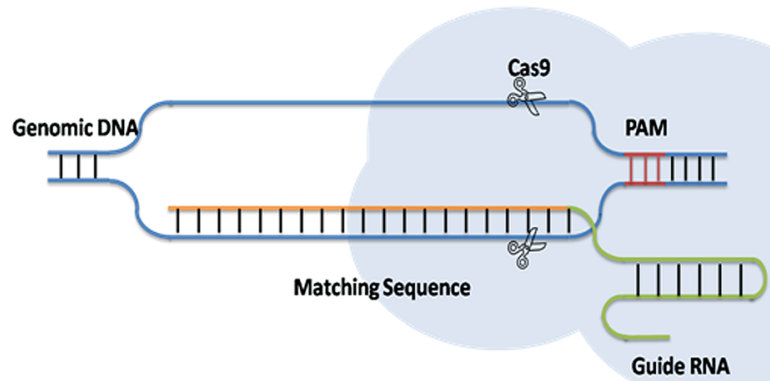

b

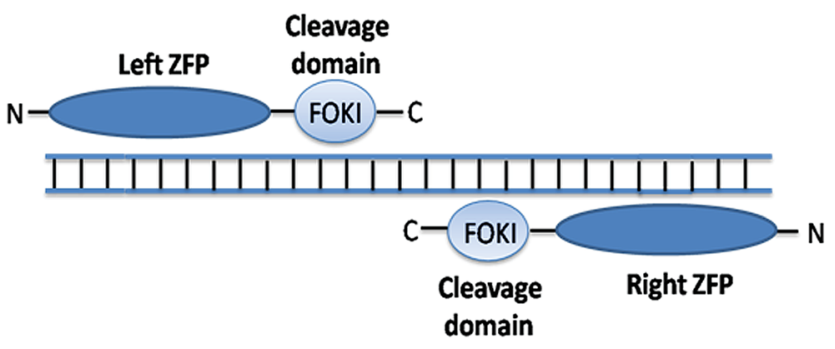

C

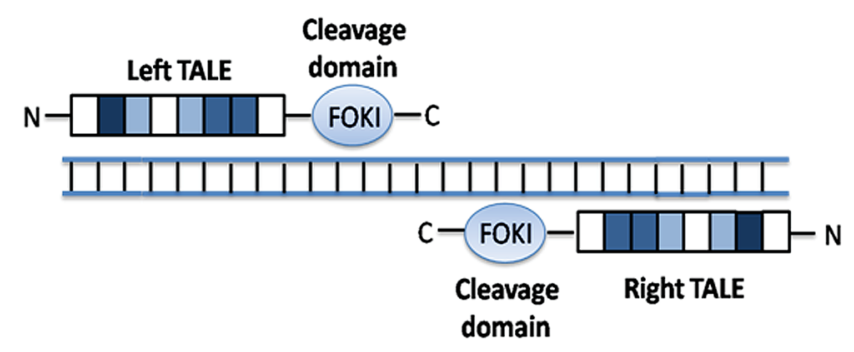

d

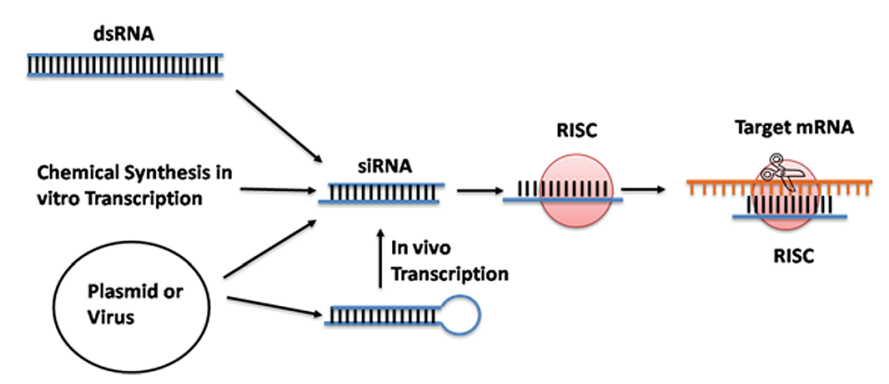

Fig. 3 Simple principles of four gene editing methods. (a) The guide RNA recognizes the PAM sequence and cleaves the Cas9 protein in front of it. (b) The ZFN identifies the target sequence and the Fok I cleavage domain cuts the DNA. (c) The TALEN identifies the target sequence and the Fok I cleavage domain cuts the DNA. (d) The dsRNA molecule enters the cells and specifically degrades the homologous mRNA. 
the treatment of cancers by targeting the molecules involved in these pathways.

\section{$2.3 \quad$ p57 in HDAC inhibitors resistance}

HDAC (histone deacetylase) is considered as the crucial epigenetic enzyme, which regulates histone acetylation and affects the target genes expression. Recently, HDAC was reported to associated with development and progression of various tumors. ${ }^{38}$ In addition, several HDAC inhibitors have been approved by FDA to treat cancers. In a recent study on pancreatic ductal adenocarcinoma of mice, Mazur et al. found that HDAC inhibitor (SAHA) induced tumor cell death by de-repression p57 gene, also known as CDKN1C. ${ }^{39}$ Further, using CRISPR-Cas9 system to delete the p57 gene, they found that p57-knockout pancreatic tumors were less responsive to the HDAC inhibitor, with reduced apoptosis. Thus, this study shows how the CRISPR-Cas9 approach is used to clarify the mechanism underlying a novel epigenetic-related therapeutic strategy.

\subsection{ASXL1 in imatinib resistance}

As a tyrosine kinase inhibitor (TKI), imatinib is widely used for treatment with chronic myelocytic leukemia (CML) patients. Similar to other TKIs, resistance is inevitable in the long-term treatment. Recently study showed ASXL1, a tumor suppressor gene which frequently mutates in CML, might be involved into imatinib resistance. Re-express ASXL1 protein using CRISPRCas9 technology in the CML cell line KBM5, which originally lacked ASXL1 expression, could result in the inhibition of cell growth, the enhancement of cell differentiation, and the increasing of drug sensitivity, ${ }^{40}$ suggesting ASXL1 would be a potential target for reversing imatinib resistance.

\subsection{Kinesin-5 A133P mutation in ispinesib resistance}

Kinesin-5 is a molecular motor protein that is essential in mitosis. Accumulation of studies have showed that kinesin-5 is involved into tumor progression and is considered as a promising target for cancer treatment. Ispinesib, an inhibitor of kinesin-5, has entered clinical trials as an anticancer agent. Recently, a CRISPR-Cas9 based screening study indicated that gene mutation of kinesin-5 A133P, but not overexpression of kinesin-12 or EGFR activation that was regarded as resistance mechanism in previous study, ${ }^{41}$ contributed to ispinesib resistance. ${ }^{42}$ This work demonstrated that drug resistance related gene mutation might be deepened by CRISPR-Cas9 system.

\section{$2.6 \mathrm{p53}$ and CTR in cisplatin resistance}

Cisplatin is a commonly used cytotoxic drug for the treatment of various tumor types, such as oesophageal adenocarcinoma (OAC). The p53 is a critical tumor suppressor and is mutated frequently in many cancers, often leading to chemoresistance and poor survival. About $70 \%$ of OACs have p53 mutations and develop resistance, so that many patients are not sensitive to long-term treatment with cisplatin, and may relapse after treatment. ${ }^{43}$ APR-246 is a first-in-class reactivator of mutant p53, which can upregulate the p53 gene, induce cell cycle arrest, inhibit cancer cell growth, and trigger apoptosis of OAC cells carrying a p53 mutation. ${ }^{44}$ Recent study showed ectopic expression of mutant p53 sensitized p53-null cells to APR-246, while p53 gene knockout diminished drug activity. Furthermore, they demonstrated that reactivation of p53 would restore chemosensitivity to a cisplatin resistant xenograft model..$^{44}$ Another investigation on the mechanisms of cisplatin resistance is related to copper transporter genes (CTR1 and CTR2) or copper chaperone genes (ATOX1 and CCS). Bompiani et al. knocked out these genes with CRISPR-Cas9 and found that the sensitivity to cisplatin did not change much compared with the untreated cells. This indicates that these genes are not necessary for the process by which cisplatin enters cells and carries out its function. ${ }^{45}$ The above studies show that CRISPR-Cas9 can be used to explore the role of specific genes in drug resistance in tumor cells, and can also be used to engineer drug-resistant cells so that they regain their sensitivity to drugs.

\subsection{MLL in epirubicin resistance}

Epirubicin is a cytotoxic agent for the treatment of bladder cancer (BC). BC is characterized by a high rate of postoperative recurrence. Studies have found that changes in the MLL gene are involved in BC recurrence. MLL encodes a transcription coactivation factor which regulates gene expression during early embryonic development and hematopoiesis. The MLL protein contains a SET (suppressor of variegation, enhancer of zeste, trithorax) domain at the C-terminus, and is a member of the MLL/trx family, which specifically methylates lysine 4 on histone $\mathrm{H} 3(\mathrm{H} 3 \mathrm{~K} 4) .{ }^{46} \mathrm{H} 3 \mathrm{~K} 4$ methylation is typically associated with transcriptionally active chromatin regions. Recent study indicated that MLL-mutant bladder cancer cells obtained using CRISPR-Cas9 technology are more resistant to epirubicin than wild-type cells. ${ }^{47}$ Thus, targeting MLL may improve the treatment of recurrent bladder cancer.

\subsection{Rsf-1 in paclitaxel resistance}

Paclitaxel is a classic drug for the treatment of lung cancer, but it readily induces resistance. ${ }^{48}$ Therefore, new ways to overcome drug resistance are urgently needed. The Rsf- 1 (remodeling and spacing factor 1) gene is located on chromosome 11 at position q13.5 and encodes a histone-binding protein. It forms an ISWI chromatin remodeling complex with hSNF2H (human sucrose nonfermenting protein 2 homologue), and this complex can participate in transcriptional regulation and chromatin remodeling. ${ }^{49}$ Rsf-1 expression is high in lung cancer and increases paclitaxel tolerance through the NF- $\kappa B$ pathway. Many other genes are also involved in drug resistance, such as genes that affect apoptosis and the cell cycle. ${ }^{50}$ Chen and colleagues knocked out Rsf-1 in lung cancer cells and xenograft mice using CRISPR-Cas9 technology, and tested whether Rsf-1 affects the sensitivity of lung cancer to paclitaxel by regulating the activation of the NF- $\mathrm{B}$ pathway and the expression of its downstream genes. The data indicated that paclitaxel reduced the ability of Rsf-1-knockout NCI-H1299 and NCI-H460 cells to migrate and proliferate, and increased apoptosis. In mice with xenograft tumors derived from Rsf-1- 
Table 1 Molecules that mediate resistance to cancer therapy agents, as identified by use of the CRISPR-Cas9 system

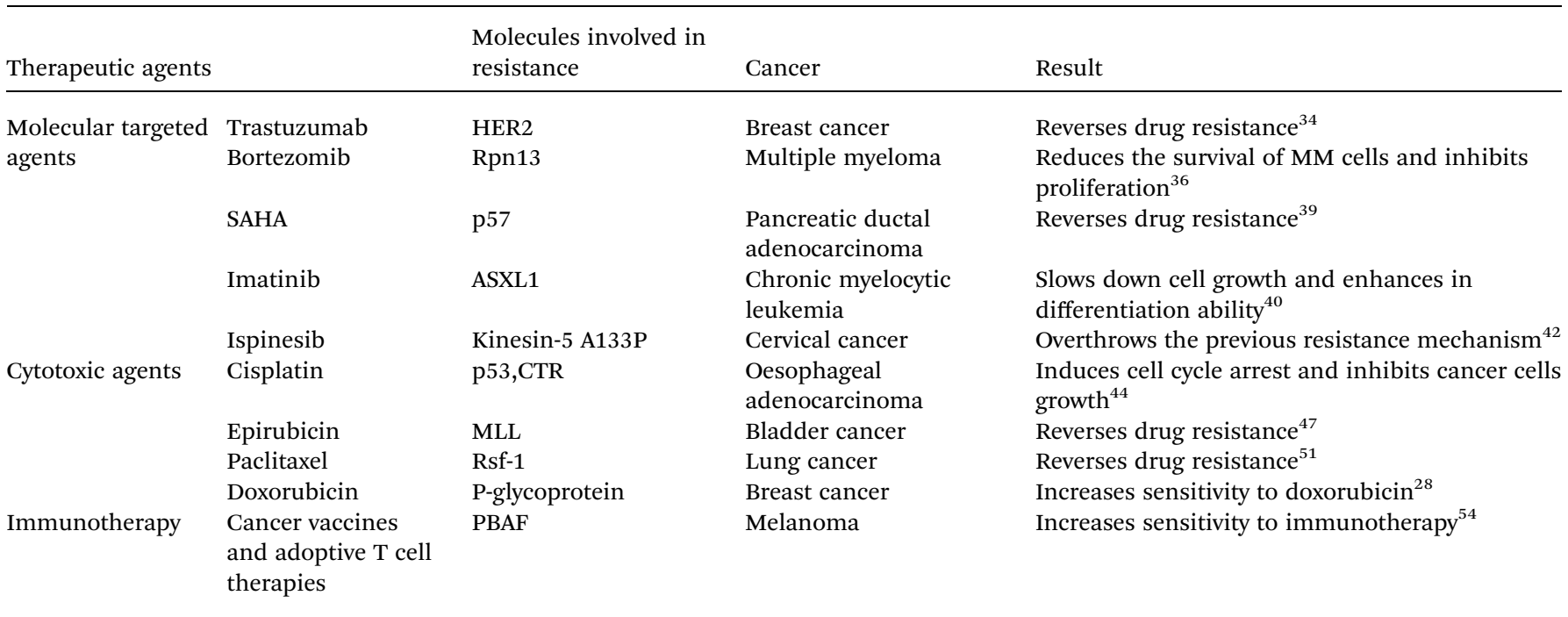

knockout cells, the anti-tumor effect of paclitaxel was enhanced. Therefore, targeting Rsf-1 by CRISPR-Cas9 technology has also become one of the approaches for treating lung cancer. ${ }^{51}$

\subsection{P-glycoprotein in doxorubicin resistance}

P-glycoprotein (P-gp) is the product of the multi-drug resistance gene (MDR1), and is considered as a classic drug resistant protein. Recently, the role of P-gp in drug resistance is also confirmed by CRISPR-Cas9 technology. Ha et al. demonstrated that knockout of MDR1 gene by transfecting the Cas9-sgRNA complex into doxorubicin-resistant breast cancer cells could enhance the sensitivity of transfected cells to doxorubicin. ${ }^{28}$ Thus, the CRISPR-Cas9 system might be a valuable and feasible method to confirm previous identified targets involved to drug resistance.

\subsection{PBAF in immunotherapy resistance}

Immunotherapy has been widely applied in the treatment of leukemia and solid tumors, and obtain a good efficacy. This therapy is usually targeted to the PD-1 receptor, which is an inhibitory receptor on $\mathrm{T}$ cells. Cytotoxic $\mathrm{T}$ cells also express $\mathrm{T}$ cell receptors which recognize peptide antigens bound to MHC class I proteins. ${ }^{52}$ In the normal immune response, this interaction leads to activation of the cytotoxic $\mathrm{T}$ cells, which find and clear abnormal cells expressing the antigen, including cancer cells. However, most cancer patients do not respond to immunotherapy, and the mechanism underlying this lack of response is poorly understood. ${ }^{53}$ To help clarify the mechanism, Pan et al. used CRISPR-Cas9 to inactivate more than 100 genes in mouse B16F10 melanoma cells, and then they tested the sensitivity of the cells to $\mathrm{T}$ cell-mediated killing. Cells lacking the genes Pbrm1, Arid2 and Brd7 were found to be more sensitive to cytotoxic T cells. These three genes encode proteins in the PBAF form of the SWI/SNF tumor suppressor complex, which regulates transcription factor recognition of chromatin. When PBAF was inactivated by CRISPR-Cas9, the B16F10 cells increased their sensitivity to interferon-gamma and their secretion of chemokines. Therefore, inactivation of the above three genes in human tumors may increase the effectiveness of PD-1 blockade, adoptive $\mathrm{T}$ cell therapies or cancer vaccines. In addition, $\mathrm{T}$ cellmediated cytotoxicity is more lethal to PBAF-deficient tumor cells. Thus, targeting the intrinsic resistance mechanisms of tumor cells may represent a new method for improving the response to immunotherapy (Table 1$)^{54}$

\section{The problems and challenges of the CRISPR-Cas9 system}

The CRISPR-Cas9 system can edit genomes accurately, it has been rapidly and widely applied to molecular biology and cell research. However, due to the fact that the specificity of CRISPRCas9 is determined by base pairing, a low targeting specificity can result in uncertain research results and the waste of manpower and material resources. Therefore, the biggest problem with the system is off-target effect. It affects the efficiency of the system, and can lead to a series of adverse consequences such as oncogene activation in the genome or other important mutations. For clinical applications and gene therapy in particular, off-target effects can cause great harm. ${ }^{55}$ In addition, the plasmids needed for CRISPR-Cas9 editing are large, and there are difficulties with the transfection process. Furthermore, the gene deletion rate is low, and even if the vectors are successfully imported into the cells, a lack of effective screening methods is also a problem. In spite of the successful generation of gene-knockout cell lines, the biological functions of cell lines formed by different monoclonal cells may not be exactly the same. This may lead to poor reproducibility in subsequent experiments with such cell lines. Moreover, the gene editing of human embryonic and germ cells has aroused strong opposition from many scientists. The rational 
application of this technology requires extensive discussions in the scientific, medical, legal and ethical communities. ${ }^{56}$

Despite these problems, CRISPR-Cas9 technology has many advantages compared with other gene editing tools, such as simple operation, high targeting efficacy and robust sequence specificity. It has a broad range of applications in site-directed genome modification. In drug-resistance research, its most important application is in making tumor cells regain their sensitivity to drugs. It is believed that with the rapid development of the technology, researchers can rapidly identify and select drug-resistance-related genes in different tumor types. The CRISPR-Cas9 system provides new ideas and a theoretical basis for figuring out drug-resistance mechanisms and for developing new treatments to overcome drug resistance. It has the potential to change all aspects of tumor biology and biomedicine. Recently CRISPR-Cpf1, as a novel class II CRISPRCas systems, is designed by an RNA-directed endonuclease, which is a smaller and simpler endonuclease than Cas9. It can overcome the limitations of some CRISPR-Cas9 systems and is expected to become a new generation of gene editing technology. Moreover, recent study showed another more sensitivity system, called specific high-sensitivity enzymatic reporter unLOCKing (SHERLOCK), ${ }^{57}$ could be used to detect tumor circulating DNA mutations, which provide a possibility to find biomarkers for diagnosis and drug resistance using circulating tumor cells or cell-free nucleic acids. ${ }^{58}$ Therefore, with the gradual application of CRISPR-Cas9 technology in clinical practice, it will be possible to achieve cancer gene therapy by carrying out genome repairs in tumor cells to correct mutations, chromosomal defects, copy number variations, and abnormal gene regulation and expression. ${ }^{59}$ In summary, the CRISPRCas9 system will provide many possibilities for overcoming drug resistance in tumors.

\section{Conflicts of interest}

There are no conflicts to declare.

\section{Acknowledgements}

This work was financially supported by National Natural Science Foundation of China (No. 81572947, 81773216), Natural Science Foundation of Liaoning Province (No. 20170540841) and Scientific Research Fund of Liaoning Provincial Education Department (No. 2017LFW01).

\section{References}

1 F. V. Karginov and G. J. Hannon, The CRISPR system: small RNA-guided defense in bacteria and archaea, Mol. Cell, 2010, 37(1), 7-19.

2 V. Kunin, R. Sorek and P. Hugenholtz, Evolutionary conservation of sequence and secondary structures in CRISPR repeats, Genome Biol., 2007, 8(4), R61.

3 I. Grissa, G. Vergnaud and C. Pourcel, The CRISPRdb database and tools to display CRISPRs and to generate dictionaries of spacers and repeats, BMC Bioinf., 2007, 8, 172.
4 Y. Ishino, H. Shinagawa, K. Makino, M. Amemura and A. Nakata, Nucleotide sequence of the iap gene, responsible for alkaline phosphatase isozyme conversion in Escherichia coli, and identification of the gene product, J. Bacteriol., 1987, 169(12), 5429-5433.

5 A. Bolotin, B. Quinquis, A. Sorokin and S. D. Ehrlich, Clustered regularly interspaced short palindrome repeats (CRISPRs) have spacers of extrachromosomal origin, Microbiology, 2005, 151(8), 2551-2561.

6 R. Barrangou, C. Fremaux, H. Deveau, M. Richards, P. Boyaval, S. Moineau, D. A. Romero and P. Horvath, CRISPR provides acquired resistance against viruses in prokaryotes, Science, 2007, 315(5819), 1709-1712.

7 J. Van der Oost, M. M. Jore, E. R. Westra, M. Lundgren and S. J. Brouns, CRISPR-based adaptive and heritable immunity in prokaryotes, Trends Biochem. Sci., 2009, 34(8), 401-407.

8 M. Jinek, K. Chylinski, I. Fonfara, M. Hauer, J. A. Doudna and E. Charpentier, A programmable dual-RNA-guided DNA endonuclease in adaptive bacterial immunity, Science, 2012, 337(6096), 816-821.

9 L. Cong, F. A. Ran, D. Cox, S. Lin, R. Barretto, N. Habib, P. D. Hsu, X. Wu, W. Jiang, L. A. Marraffini and F. Zhang, Multiplex genome engineering using CRISPR/Cas systems, Science, 2013, 339(6121), 819-823.

10 S. H. Sternberg, B. LaFrance, M. Kaplan and J. A. Doudna, Conformational control of DNA target cleavage by CRISPRCas9, Nature, 2015, 527(7576), 110-113.

11 S. C. Knight, L. Xie, W. Deng, B. Guglielmi, L. B. Witkowsky, L. Bosanac, E. T. Zhang, M. El Beheiry, J. B. Masson, M. Dahan, Z. Liu, J. A. Doudna and R. Tjian, Dynamics of CRISPR-Cas9 genome interrogation in living cells, Science, 2015, 350(6262), 823-826.

12 I. M. Slaymaker, L. Gao, B. Zetsche, D. A. Scott, W. X. Yan and F. Zhang, Rationally engineered Cas9 nucleases with improved specificity, Science, 2016, 351(6268), 84-88.

13 K. E. Savell and J. J. Day, Applications of CRISPR/Cas9 in the Mammalian Central Nervous System, Yale J. Biol. Med., 2017, 90(4), 567-581.

14 W. Jiang and L. A. Marraffini, CRISPR-Cas: New Tools for Genetic Manipulations from Bacterial Immunity Systems, Annu. Rev. Microbiol., 2015, 69, 209-228.

15 C. R. Hale, P. Zhao, S. Olson, M. O. Duff, B. R. Graveley, L. Wells, R. M. Terns and M. P. Terns, RNA-guided RNA cleavage by a CRISPR RNA-Cas protein complex, Cell, 2009, 139(5), 945-956.

16 M. Babu, N. Beloglazova, R. Flick, C. Graham, T. Skarina, B. Nocek, A. Gagarinova, O. Pogoutse, G. Brown, A. Binkowski, S. Phanse, A. Joachimiak, E. V. Koonin, A. Savchenko, A. Emili, J. Greenblatt, A. M. Edwards and A. F. Yakunin, A dual function of the CRISPR-Cas system in bacterial antivirus immunity and DNA repair, Mol. Microbiol., 2011, 79(2), 484-502.

17 K. S. Makarova, Y. I. Wolf, O. S. Alkhnbashi, F. Costa, S. A. Shah, S. J. Saunders, R. Barrangou, S. J. Brouns, E. Charpentier, D. H. Haft, P. Horvath, S. Moineau, F. J. Mojica, R. M. Terns, M. P. Terns, M. F. White, 
A. F. Yakunin, R. A. Garrett, J. van der Oost, R. Backofen and E. V. Koonin, An updated evolutionary classification of CRISPR-Cassystems, Nat. Rev. Microbiol., 2015, 13(11), 722736.

18 M. E. Zegans, J. C. Wagner, K. C. Cady, D. M. Murphy, J. H. Hammond and G. A. O'Toole, Interaction between bacteriophage DMS3 and host CRISPR region inhibits group behaviors of Pseudomonas aeruginosa, J. Bacteriol., 2009, 191(1), 210-219.

19 P. Viswanathan, K. Murphy, B. Julien, A. G. Garza and L. Kroos, Regulation of dev, an operon that includes genes essential for Myxococcus xanthus development and CRISPRassociated genes and repeats, J. Bacteriol., 2007, 189(10), 3738-3750.

20 J. E. DiCarlo, J. E. Norville, P. Mali, X. Rios, J. Aach and G. M. Church, Genome engineering in Saccharomyces cerevisiae using CRISPR-Cassystems, Nucleic Acids Res., 2013, 41(7), 4336-4343.

21 G. Gasiunas, R. Barrangou, P. Horvath and V. Siksnys, Cas9crRNA ribonucleoprotein complex mediates specific DNA cleavage for adaptive immunity in bacteria, Proc. Natl. Acad. Sci. U. S. A., 2012, 109(39), E2579-E2586.

22 Y. G. Kim, J. Cha and S. Chandrasegaran, Hybrid restriction enzymes: zinc finger fusions to Fok I cleavage domain, Proc. Natl. Acad. Sci. U. S. A., 1996, 93(3), 1156-1160.

23 T. Gaj, C. A. Gersbach and R. C. Barbas, ZFN, TALEN, and CRISPR/Cas-based methods for genome engineering, Trends Biotechnol., 2013, 31(7), 397-405.

24 D. Hockemeyer, H. Y. Wang, S. Kiani, C. S. Lai, Q. Gao, J. P. Cassady, G. J. Cost, L. Zhang, Y. Santiago, J. C. Miller, B. Zeitler, J. M. Cherone, X. D. Meng, S. J. Hinkley, E. J. Rebar, P. D. Gregory, F. D. Urnov and R. Jaenisch, Genetic engineering of human pluripotent cells using TALE nucleases, Nat. Biotechnol., 2011, 29(8), 731-734.

25 Q. W. Shan, Y. P. Wang, K. L. Chen, Z. Liang, J. Li, Y. Zhang, K. Zhang, J. X. Liu, D. F. Voytas, X. L. Zheng, Y. Zhang and C. X. Gao, Rapid and efficient gene modification in rice and Brachypodium using TALENs, Mol. Plant, 2013, 6(4), 1365-1368.

$26 \mathrm{~S}$. Guo and K. J. Kemphues, par-1, a gene required for establishing polarity in C. elegans embryos, encodes a putative Ser/Thr kinase that is asymmetrically distributed, Cell, 1995, 81(4), 611-620.

27 C. Lipardi, Q. Wei and B. M. Paterson, RNAi as random degradative PCR: siRNA primers convert mRNA into dsRNAs that are degraded to generate new siRNAs, Cell, 2001, 107(3), 297-307.

28 J. S. Ha, J. Byun and D. R. Ahn, Overcoming doxorubicin resistance of cancer cells by Cas9-mediated gene disruption, Sci. Rep., 2016, 6, 22847.

29 S. J. Kim, J. Kyung Hee, S. Mi Kwon, L. Joo Han and H. SoonSun, Synergistic anticancer effects through tumor vessel normalization by PI3K inhibitors, Eur. J. Cancer, 2016, 69, S129.

30 F. Wang, T. Guo, H. Jiang, et al., A comparison of CRISPR/ Cas9 and siRNA-mediated ALDH2 gene silencing in human cell lines, Mol. Genet. Genomics, 2018, 293(3), 1-15.
31 K. C. Wiegand, A. F. Lee, O. M. Al-Agha, et al., Loss of BAF250a (ARID1A) is frequent in high-grade endometrial carcinomas, J. Pathol., 2011, 224(3), 328.

32 S. Jones, T. L. Wang, S. Iem, et al., Frequent mutations of chromatin remodeling gene ARID1A in ovarian clear cell carcinoma, Science, 2010, 330(6001), 228-231.

33 R. Belvedere, V. Bizzarro, A. Popolo, et al., Role of intracellular and extracellular annexin A1 in migration and invasion of human pancreatic carcinoma cells, $B M C$ Cancer, 2014, 14(1), 961.

34 K. Berns, A. Sonnenblick, A. Gennissen, et al., Loss of ARID1A activates ANXA1, which serves as a predictive biomarker for trastuzumab resistance, Clin. Cancer Res., 2016, 22(21), 5238.

35 P. Richardson, S. Jagannath, N. Raje, et al., A phase 2 trial of lenalidomide, bortezomib, and dexamethasone in patients with relapsed and relapsed/refractory myeloma, Blood, 2014, 123(10), 1461-1469.

36 Y. Song, A. Ray, S. Li, et al., Targeting proteasome ubiquitin receptor Rpn13 in multiple myeloma, Leukemia, 2016, 30(9), 1877.

37 Z. Chen, A. E. Teo and N. McCarty, ROS-Induced CXCR4 Signaling Regulates Mantle Cell Lymphoma (MCL) Cell Survival and Drug Resistance in the Bone Marrow Microenvironment via Autophagy, Clin. Cancer Res., 2016, 22(1), 187-199.

38 A. C. West and R. W. Johnstone, New and emerging HDAC inhibitors for cancer treatment, J. Clin. Invest., 2014, 124(1), 30-39.

39 P. K. Mazur, A. Herner, S. S. Mello, et al., Combined inhibition of BET family proteins and histone deacetylases as a potential epigenetics-based therapy for pancreatic ductal adenocarcinoma, Nat. Med., 2015, 21(10), 1163-1171.

40 V. Simona, D. Hamid, B. Matthias, et al., ASXL1mutation correction by CRISPR/Cas9 restores gene function in leukemia cells and increases survival in mouse xenografts, OncoTargets Ther., 2015, 6(42), 44061-44071.

$41 \mathrm{M}$. Ono and M. Kuwano, Molecular mechanisms of epidermal growth factor receptor (EGFR) activation and response to gefitinib and other EGFR-targeting drugs, Clin. Cancer Res., 2006, 12(24), 7242-7251.

42 C. Kasap, O. Elemento and T. M. Kapoor, DrugTargetSeqR: a genomics- and CRISPR-Cas9-based method to analyze drug targets, Nat. Chem. Biol., 2014, 10(8), 626-628.

43 J. L. Raoul, Definitive chemoradiotherapy with FOLFOX versus fluorouracil and cisplatin in patients with oesophageal cancer (PRODIGE5/ACCORD17): final results of a randomised, phase 2/3 trial, Lancet Oncol., 2014, 15(3), 305-314.

44 D. S. H. Liu, M. Read, C. Cullinane, et al., APR-246 potently inhibits tumour growth and overcomes chemoresistance in preclinical models of oesophageal adenocarcinoma, Gut, 2015, 64(10), 1506-1516.

45 K. M. Bompiani, C. Y. Tsai, F. P. Achatz, et al., Copper transporters and chaperones CTR1, CTR2, ATOX1, and CCS as determinants of cisplatin sensitivity, Metallomics, 2016, 8(9), 951-962. 
46 J. U. Marquardt and S. S. Thorgeirsson, Linking MLL and the HGF-MET signaling pathway in liver cancer, J. Clin. Invest., 2013, 123(7), 2780-2783.

$47 \mathrm{~S} . \mathrm{Wu}, \mathrm{Z}$. Yang, R. Ye, et al., ovel variants in MLL confer to bladder cancer recurrence identified by whole-exome sequencing, OncoTargets Ther., 2016, 7(3), 2629-2645.

48 Y. Liu, X. Wu, Y. Sun, et al., Silencing of X-linked inhibitor of apoptosis decreases resistance to cisplatin and paclitaxel but not gemcitabine in non-small cell lung cancer, J. Int. Med. Res., 2011, 39(5), 1682-1692.

49 J. J. Sheu, B. Guan, J. H. Choi, et al., Rsf-1, a Chromatin Remodeling Protein, Induces DNA Damage and Promotes Genomic Instability, J. Biol. Chem., 2010, 285(49), 3826038269.

50 J. H. Choi, J. J. Sheu, B. Guan, et al., Functional analysis of 11q13.5 amplicon identifies Rsf-1 (HBXAP) as a gene involved in paclitaxel resistance in ovarian cancer, Cancer Res., 2009, 69(4), 1407-1415.

51 X. Chen, X. Sun, J. Guan, et al., Rsf-1 Influences the Sensitivity of Non-Small Cell Lung Cancer to Paclitaxel by Regulating NF- $\kappa \mathrm{B}$ Pathway and Its Downstream Proteins, Cell. Physiol. Biochem., 2017, 44(6), 2322.

52 N. Zhang and M. J. Bevan, CD8(+) T cells: foot soldiers of the immune system, Immunity, 2011, 35(2), 161-168.
53 P. Sharma, S. Hu-Lieskovan, J. A. Wargo and A. Ribas, Primary, Adaptive, and Acquired Resistance to Cancer Immunotherapy, Cell, 2017, 168(4), 707-723.

54 D. Pan, A. Kobayashi, P. Jiang, et al., A major chromatin regulator determines resistance of tumor cells to $\mathrm{T}$ cellmediated killing, Science, 2018, 359(6377), 770-775.

55 V. Pattanayak, S. Lin, J. P. Guilinger, et al., High-throughput profiling of off-target DNA cleavage reveals RNAprogrammed Cas9 nuclease specificity, Nat. Biotechnol., 2013, 31(9), 839-843.

56 D. Baltimore, P. Berg, M. Botchan, et al., A prudent path forward for genomic engineering and germline gene modification, Science, 2015, 348(6230), 36.

57 J. S. Gootenberg, O. O. Abudayyeh, J. W. Lee, et al., Nucleic acid detection with CRISPR-Cas13a/C2c2, Science, 2017, 356(6336), 438-442.

58 J. S. Gootenberg, O. O. Abudayyeh, J. W. Lee, et al., Nucleic acid detection with CRISPR-Cas13a/C2c2, Science, 2017, 356(6336), 438-442.

59 D. A. Scott and F. Zhang, Implications of human genetic variation in CRISPR-based therapeutic genome editing, Nat. Med., 2017, 23(9), 1095. 